\title{
Influência das atividades antrópicas na qualidade das águas da bacia hidrográfica do Rio Salitre ${ }^{1}$
}

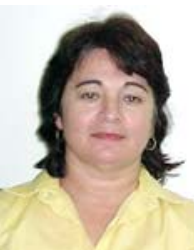

Luiza T. de L. Brito ${ }^{2}$, Vajapeyam S. Srinivasan ${ }^{3}$, Aderaldo de S. Silva ${ }^{4}$, Hans R. Gheyi ${ }^{3}$, Carlos de O. Galvão ${ }^{3}$ \& Luiz C. Hermes ${ }^{4}$

${ }_{1}^{1}$ Parte da Tese de Doutorado do primeiro autor, apresentada à Universidade Federal de Campina Grande. ${ }^{2}$ EMBRAPA Semi-Árido, CP 23, Petrolina, PE. CEP 56300-970. Fone: (87) 3862-1711, E-mail: luizatlb@cpatsa.embrapa.br

${ }^{3}$ UFCG, CEP 58109-970. Campina Grande, PB. Fone: (83) 310-1157. E-mail:srinivas@rechid.ufcg.edu.br; hans@deag.ufcg.edu.br; galvao@dec.ufcg.edu.br

${ }^{4}$ EMBRAPA Meio Ambiente, CP 29. Jaguariúna, SP. Fone: (19) 3867-8700. E-mail: aderaldo@cnpma.embrapa.br; hermes@cnpma.embrapa.br

Protocolo 13 - 22/7/2004 - Aprovado em 15/4/2005

Resumo: Avaliar os impactos das atividades agrícolas sobre a qualidade das águas da bacia hidrográfica do Rio Salitre, foi o objetivo deste estudo. Para isto, 92 fontes hídricas localizadas em áreas irrigadas ou não, foram analisadas nos períodos de chuva e sem chuva de 2001, avaliando-se os principais indicadores para consumo humano e irrigação. A partir dos resultados, observou-se a influência da irrigação sobre a qualidade das águas, ocorrendo aumentos significativos na condutividade elétrica da água e no extrato de saturação dos solos; esses aumentos indicam baixa eficiência dos sistemas de produção, constatando-se que, em média, 35 e $77 \%$ das fontes hídricas foram classificadas como $\mathrm{C}_{3}$ e $\mathrm{C}_{4}$, respectivamente; também, que $78 \%$ dessas fontes estão localizadas sob formações calcárias, cuja influência sobre a qualidade das águas pode ser notada pelos elevados teores de sólidos dissolvidos totais (SDT = 7.940,0; $5.060,0 \mathrm{mg} \mathrm{L}^{-1}$ ), relação de adsorção de sódio (RAS $\left.=25,27 ; 6,71 \mathrm{mmol} \mathrm{L}^{-1}\right)^{1 / 2}$ ), dureza total (DT $=2.999,6 ; 2.940,0 \mathrm{mg} \mathrm{L}^{-1}$ ) e percentual de cloretos (CIP $=97,51 ; 94,59 \%$ ), entre outros, limitando sua utilização; tais resultados apontam para a necessidade de medidas de preservação e de conservação dos recursos hídricos e dos solos desta bacia hidrográfica para permitir a sustentabilidade ambiental.

Palavras-chave: impactos ambientais, irrigação, recursos hídricos, semi-árido

\section{Influence of anthropic activities on water quality of Salitre river basin}

\begin{abstract}
This study had the main objective of evaluating the impacts of agricultural activities on water quality of Salitre watershed in the brazilian semi-arid region. For that, 92 water sources, located in areas with and without the influence of irrigation, were analyzed during rainy and dry seasons in 2001, evaluating the main parameters of water quality for human consumption and irrigation. From the results the influence of irrigation on water quality was observed, with the occurrences of significant increases of electrical conductivity of the waters and saturation extract of the soil; increases in the parameters indicate low efficiency of farming systems, 35 and $77 \%$ of the water sources were classified as $\mathrm{C}_{3}$ and $\mathrm{C}_{4}$, respectively. Also that $78 \%$ are located under calcareous formations and its influence on water quality can be observed from the high total dissolved solids (TDS $=7,940.0 ; 5,060.0 \mathrm{mg} \mathrm{L}^{-1}$ ), sodium adsorption ratio $\mathrm{SAR}=25.27$; $6.71\left(\mathrm{mmol} \mathrm{L}^{-1}\right)^{1 / 2}$, total hardness $\left(\mathrm{TH}=2,999.6 ; 2,940.0 \mathrm{mg} \mathrm{L}^{-1}\right)$, chloride percentage (CIP $=$ $97.51 ; 94.59 \%)$, among other parameters, limiting their utilization; these results point out the need for strategies of preservation and conservation of the water resources and soils of the Salitre watershed in order to guarantee its sustainability.
\end{abstract}

Key words: environmental impacts, irrigation, water resources, semi-arid 


\section{INTRODUÇÃO}

$\mathrm{O}$ consumo mundial de água aumentou mais de seis vezes em menos de um século, mais que o dobro das taxas de crescimento da população. Em nível global, os recursos hídricos tendem a se tornar mais escassos, devido aos processos de uso e de poluição crescentes, caso não haja ações enérgicas visando à melhoria da gestão da oferta e da demanda da água (Freitas \& Santos, 1999). De acordo com Brown et al. (2000) a escassez de água refletirá na produção de alimentos, uma vez que são necessárias 1000 toneladas de água para produzir uma tonelada de grãos.

Estima-se que o Brasil detém 13,8\% da disponibilidade hídrica mundial; entretanto, apresenta uma distribuição interregional bastante heterogênea, tanto em termos de disponibilidade quanto de qualidade das águas (Freitas \& Santos, 1999). No semi-árido brasileiro e mais especificamente na bacia hidrográfica do Rio Salitre, esta situação não é diferente; fatores climáticos, geológicos e antrópicos influenciam na renovação das reservas hídricas e na variação da qualidade das águas. Dada a predominância do substrato cristalino, os aqüíferos são de baixa produtividade, os poços são rasos e apresentam vazões inferiores a $3,0 \mathrm{~m}^{3} \mathrm{~h}^{-1}$, elevados teores de sólidos dissolvidos totais, em média, 3,0 $\mathrm{g} \mathrm{L}^{-1}$ (Leal, 1999).

No contexto da influência das atividades antrópicas na qualidade das águas, a agricultura é tida como a principal consumidora e uma das principais poluidoras dos recursos hídricos, sendo a salinidade e a contaminação por nitrato os principais indicadores de poluição das águas (Ongley, 2001; Brown et al., 2000). Segundo Resende (2002), sob determinadas condições de solo e clima e o uso excessivo ou o manejo inadequado de fertilizantes, podem acarretar o enriquecimento das fontes hídricas, promovendo a eutrofização de suas águas, com sérios prejuízos ao ambiente e à própria saúde humana.

Para melhor entendimento das interações entre os fatores de produção agrícola, Girardin et al. (2000) desenvolveram uma metodologia para verificar se os princípios da sustentabilidade estavam sendo seguidos, avaliando-se nove indicadores agroecológicos, concluindo que a variabilidade dos sistemas de produção impõe estudos específicos para cada propriedade, surgindo a necessidade da inclusão de outros indicadores.

De modo geral, a qualidade da água é definida por sua composição física, química, biológica e radioativa e, conseqüentemente, pelos efeitos que seus constituintes podem causar ao ambiente. Considerados nobres, determinados usos exigem rigoroso controle de qualidade das águas; além disso, padrões de qualidade para consumo humano, indústria, irrigação, variam enormemente. Em função de seus usos e se considerando suas características, vários organismos estabeleceram normas e padrões específicos de qualidade da água. No Brasil, as normas de qualidade de água para consumo humano são regidas pelo Ministério da Saúde, que as delibera para instituições competentes, por meio da Portaria $\mathrm{n}^{\circ} .518$, de 25 de março de 2004 (Brasil, 2004).

$\mathrm{Na}$ bacia hidrográfica do Rio Salitre, estado da Bahia, as sub-regiões de Caatinga do Moura (Jacobina), Taquarandi (Mirangaba) e Juazeiro, têm como principal atividade econômica a agricultura irrigada, cultivando principalmente hortifrutícolas para atender aos mercados interno e externo; nessas áreas pratica-se uma diversidade de sistemas de produção, a maioria de baixa eficiência de aplicação da água de irrigação e com inadequado manejo dos solos e do uso de fertilizantes e defensivos favorecendo, conseqüentemente, a poluição das águas; assim, objetivou-se, neste estudo, avaliar os impactos das atividades agrícolas sobre a qualidade das águas da bacia hidrográfica do Rio Salitre, visando definir medidas de preservação e conservação dos recursos hídricos.

\section{MATERIAL E MÉTODOS}

\section{Caracterização geral da bacia hidrográfica do Rio Salitre}

A bacia hidrográfica do Rio Salitre inicia-se em Morro do Chapéu, BA, na Chapada Diamantina, e deságua no Rio São Francisco, no município de Juazeiro; está compreendida entre as latitudes $9^{\circ} 27^{\prime}$ e $17^{\circ} 14^{\prime}$ e as longitudes $40^{\circ} 22^{\prime}$ e $41^{\circ} 30^{\prime}$, em uma área de $13.200 \mathrm{~km}^{2}$ e comprimento do curso principal de $270 \mathrm{~km}$, aproximadamente. O clima predominante é do tipo BSh'W', segundo a classificação de Köppen, ou seja, clima semi-árido, cujo microclima oscila de úmido a subúmido, no município de Mirangaba. As precipitações médias anuais variam de 400 a $800 \mathrm{~mm}$, concentradas nos meses de janeiro a abril (Bahia, 1986; SEI, 1999).

Estudos realizados por Bahia (1986) e Neves (1972) indicam a predominância de solos Cambissolos eutróficos, Latossolos Vermelho Amarelo, Litólicos, Planossolos, Bruno não Cálcicos, Podzólicos Vermelho Amarelo, Areias Quartzosas e Vertissolos, entre outras pequenas manchas. Também, as formações dos grupos Chapada Diamantina e Bambuí, seguidos dos Calcários Caatinga e de pequenas manchas dos grupos Jacobina, Cabrobó e Salitre, na extremidade norte da bacia. No grupo Chapada Diamantina predominam os metarenitos intercalados com argilas e se subdivide nas formações Tombador, Caboclo e Morro do Chapéu. O grupo Bambuí é constituído por canais e fendas formados a partir da dissolução das rochas, condicionando a formação de aqǘfero livre; enfim, subdividese em Formação Bebedouro e Formação Salitre e ocorrem na parte central da bacia.

\section{Caracterização das águas superficiais e subterrâneas}

Foram coletadas amostras de água em fontes naturais, superficiais e subterrâneas, em março e dezembro de 2001, caracterizando os períodos de chuva (PC) e sem chuva (PV), no total de 92 fontes hídricas representativas da bacia. As amostras de água foram armazenadas em garrafas plásticas, com capacidade para $1 / 2$ litro, vedadas, identificadas e devidamente acondicionadas em caixa de isopor com gelo, até o Laboratório de Solos e Água, da Embrapa Semi-Árido, em Petrolina, PE, como recomendado por Claessen (1997). Analisaram-se as principais concentrações iônicas, como cálcio $\left(\mathrm{Ca}^{++}\right)$, magnésio $\left(\mathrm{Mg}^{++}\right)$, sódio $\left(\mathrm{Na}^{+}\right)$, potássio $\left(\mathrm{K}^{+}\right)$, carbonato $\left(\mathrm{CO}_{3}{ }^{-}\right)$, bicarbonato $\left(\mathrm{HCO}_{3}{ }^{-}\right)$, cloreto $(\mathrm{Cl}-)$, sulfato $\left(\mathrm{SO}_{4}{ }^{-}\right)$e ferro $\left(\mathrm{Fe}^{++}\right)$; também, foram feitas avaliações da qualidade das águas em tempo real, utilizando-se sondas multiparâmetros, para as variáveis: temperatura $(\mathrm{T}), \mathrm{pH}$, condutividade elétrica $(\mathrm{CE})$, sólidos dissolvidos totais (SDT), oxigênio dissolvido (OD) e amônio $\left(\mathrm{NH}_{4}^{+}-\mathrm{N}\right)$, visando determinar os indicadores 
relacionados aos riscos de salinidade e sodicidade aos solos e toxicidade às culturas, pela presença de cloretos e dureza total, como recomendado em Ayers \& Westcot (1991) e Pizarro (1978).

Coletaram-se nas áreas irrigadas, amostras compostas de solos para caracterização físico-química, avaliando-se a condutividade elétrica do extrato de saturação $\left(\mathrm{CE}_{\mathrm{s}}\right), \mathrm{pH}$, matéria orgânica (M.O), fósforo total $\left(\mathrm{P}_{\mathrm{T}}^{+++}\right)$, potássio $\left(\mathrm{K}^{+}\right)$, cálcio $\left(\mathrm{Ca}^{++}\right)$, magnésio $\left(\mathrm{Mg}^{++}\right)$, sódio $\left(\mathrm{Na}^{+}\right)$, acidez potencial $(\mathrm{H}+\mathrm{Al})$ e porcentagem de sódio trocável (PST), analisadas no Laboratório de Solos e Água, da Embrapa Semi-Árido, em Petrolina, PE, como recomendado por Claessen (1997).

\section{RESULTADOS E DISCUSSÃO}

A partir dos resultados obtidos, pode-se observar grande variabilidade nas variáveis analisadas, refletida pelos indicadores apresentados na Tabela 1. Para as fontes hídricas superficiais, os sólidos dissolvidos totais (SDT) variaram de 30 a $7.940,0 \mathrm{mg} \mathrm{L}^{-1}$ (CANTI e LBRANCA), em apenas quatro fontes esses valores foram superiores ao limite recomendado pela legislação que estabelece normas e padrões de qualidade de água para consumo humano $\left(1.000 \mathrm{mg} \mathrm{L}^{-1}\right)$, de acordo com a Portaria 518, do Ministério da Saúde (Brasil, 2004). De forma diferente, as águas subterrâneas, principal fonte hídrica destinada ao consumo doméstico, embora também apresentem grande variabilidade nos valores dos sólidos dissolvidos totais (LBAT1: SDT $=40 \mathrm{mg} \mathrm{L}^{-1}$ a BARRET1: SDT 5.370,0 $\mathrm{mg} \mathrm{L}^{-1}$ ), em $50 \%$ destas, os valores dos SDT foram superiores ao limite recomendado (Figura 1). A Figura 1 apresenta a localização das fontes hídricas superficiais e subterrâneas analisadas.

Variabilidade semelhante também foi observada na dureza total (DT) das águas, tanto para as fontes superficiais quanto

Tabela 1. Índices de qualidade das águas para as fontes hídricas superficiais e subterrâneas analisadas nos períodos de chuvas (PC) e sem chuvas (PV) de 2001

\begin{tabular}{|c|c|c|c|c|c|c|c|c|c|c|c|c|c|c|c|c|c|}
\hline \multirow[t]{2}{*}{$\begin{array}{l}\mathrm{N}^{\mathrm{o}} \mathrm{da} \\
\text { fonte }^{*}\end{array}$} & \multicolumn{2}{|c|}{$\mathrm{pH}$} & \multicolumn{2}{|c|}{$\operatorname{SDT}\left(\mathrm{mg} \mathrm{L}^{-1}\right)$} & \multicolumn{2}{|c|}{$\begin{array}{c}\text { RAS } \\
\left(\mathrm{mmol} \mathrm{L}^{-1}\right)^{1 / 2}\end{array}$} & \multicolumn{2}{|c|}{$\begin{array}{c}\mathrm{DT} \\
\left(\mathrm{mg} \mathrm{L}^{-1}\right)\end{array}$} & \multirow[t]{2}{*}{$\begin{array}{l}\mathrm{N}^{\mathrm{o}} \mathrm{da} \\
\text { fonte }^{*}\end{array}$} & \multicolumn{2}{|c|}{$\mathrm{pH}$} & \multicolumn{2}{|c|}{$\begin{array}{c}\mathrm{SDT} \\
\left(\mathrm{mg} \mathrm{L}^{-1}\right)\end{array}$} & \multicolumn{2}{|c|}{$\begin{array}{c}\text { RAS } \\
\left(\mathrm{mmol} \mathrm{L}^{-1}\right)^{1 / 2}\end{array}$} & \multicolumn{2}{|c|}{$\begin{array}{c}\mathrm{DT} \\
\left(\mathrm{mg} \mathrm{L}^{-1}\right)\end{array}$} \\
\hline & $\mathrm{PC}$ & PV & $\mathrm{PC}$ & PV & $\mathrm{PC}$ & PV & $\mathrm{PC}$ & PV & & $\mathrm{PC}$ & PV & $\mathrm{PC}$ & PV & $\mathrm{PC}$ & PV & $\mathrm{PC}$ & PV \\
\hline 1 & 3,58 & 4,80 & 100,0 & 49,0 & 1,5 & 1,30 & 19,9 & 1,30 & 47 & 7,76 & 1,50 & 640,0 & 759,0 & 1,34 & 1,53 & 419,8 & 360,0 \\
\hline $2^{* *}$ & 8,00 & - & 2390,0 & - & 4,9 & - & 829,5 & - & $48^{* *}$ & - & 7,50 & - & 190,0 & - & 0,52 & - & 124,9 \\
\hline 3 & 8,29 & 7,70 & 507,0 & 698,0 & 2,5 & 9,52 & 234,9 & 104,9 & 49 & 5,67 & 6,40 & 40,0 & 45,0 & 0,98 & 0,60 & 24,9 & 20,0 \\
\hline 4 & 7,54 & 6,90 & 398,0 & 114,0 & 5,9 & 2,12 & 64,9 & 25,0 & 50 & 7,44 & 7,30 & 230,0 & 215,0 & 0,53 & 0,45 & 154,8 & 165,0 \\
\hline 5 & 8,95 & 6,20 & 60,0 & 314,0 & 0,53 & 3,65 & 29,9 & 55,0 & 51 & 7,81 & 7,60 & 240,0 & 241,0 & 0,52 & 0,54 & 174,9 & 204,9 \\
\hline 6 & 8,88 & 6,20 & 770,0 & 54,0 & 0,16 & 0,19 & 69,9 & 35,0 & 52 & 7,08 & 7,20 & 1800,0 & 2071,0 & 2,00 & 4,20 & 1004,4 & 1069,6 \\
\hline 7 & 7,25 & 5,70 & 175,0 & 283,0 & 4,40 & 7,94 & 24,9 & 25,0 & 53 & 7,58 & 7,40 & 1340,0 & 2239,0 & 2,68 & 4,37 & 959,2 & 1024,4 \\
\hline 8 & 8,15 & 7,30 & 228,0 & 430,0 & 0,20 & 1,82 & 159,9 & 70,0 & 54 & 7,56 & 7,50 & 2050,0 & 2029,0 & 2,58 & 4,47 & 954,6 & 959,8 \\
\hline 9 & 7,99 & 6,80 & 99,00 & 221,0 & 0,30 & 0,89 & 49,9 & 60,0 & 55 & 7,84 & 7,20 & 1080,0 & 1879,0 & 3,41 & 3,42 & 464,9 & 874,6 \\
\hline 10 & 6,35 & 7,80 & 230,0 & 271,0 & 1,17 & 1,83 & 29,9 & 120,0 & 56 & 8,50 & 8,30 & 240,0 & 301,0 & 0,65 & 0,89 & 149,9 & 155,0 \\
\hline 11 & 5,08 & 6,10 & 30,0 & 30,0 & 0,57 & 0,65 & 34,9 & 20,0 & $57^{* *}$ & 7,87 & 7,90 & 50,0 & - & 1,04 & - & 24,9 & 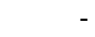 \\
\hline 12 & 4,23 & 3,40 & 50,0 & 53,0 & 1,08 & 0,96 & 14,9 & 20,0 & 58 & 7,41 & 7,60 & 430,0 & 399,0 & 1,71 & 1,79 & 239,9 & 169,9 \\
\hline 13 & 7,75 & 7,60 & 1868,0 & 5046,0 & 3,70 & 2,15 & 849,7 & 2999,6 & 59 & 7,32 & 7,80 & 2050,0 & 1922,0 & 3,85 & 6,29 & 839,9 & 789,8 \\
\hline 14 & 8,08 & 7,40 & 923,0 & 1317,0 & 2,69 & 3,90 & 459,8 & 610,0 & 60 & 8,04 & 7,50 & 1080,0 & 625,0 & 5,29 & 4,88 & 159,9 & 190,0 \\
\hline $15^{* *}$ & 7,98 & - & 1063,0 & - & 2,95 & - & 494,9 & - & 61 & 7,30 & 7,80 & 1050,0 & 1028,0 & 2,87 & 3,08 & 529,9 & 455,0 \\
\hline $16^{* *}$ & 8,30 & - & 1031,0 & - & 2,99 & - & 499,9 & - & 62 & 7,77 & 7,80 & 1050,0 & 1055,0 & 2,41 & 1,72 & 469,8 & 524,8 \\
\hline 17 & 7,20 & 7,40 & 890,0 & 952,0 & 1,56 & 1,18 & 519,9 & 530,0 & $63^{* *}$ & - & 7,60 & - & 286,0 & - & 1,72 & - & 124,9 \\
\hline 18 & 7,18 & 7,70 & 654,0 & 664,0 & 1,35 & 1,00 & 279,9 & 344,9 & 64 & 7,70 & 7,10 & 300,0 & 627,0 & 0,59 & 2,85 & 199,9 & 224,9 \\
\hline 19 & 7,57 & 7,60 & 690,0 & 694,0 & 1,32 & 0,48 & 314,9 & 355,0 & $65^{* *}$ & 7,31 & - & 150,0 & - & 0,76 & - & 94,9 & 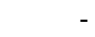 \\
\hline 20 & 7,99 & 8,00 & 1854,0 & 2199,0 & 4,04 & 4,87 & 759,8 & 865,3 & 66 & 7,91 & 8,00 & 620,0 & 714,0 & 2,27 & 3,36 & 324,0 & 220,0 \\
\hline 21 & 7,86 & 7,70 & 280,0 & 271,0 & 0,54 & 0,05 & 204,9 & 200,0 & 67 & 7,27 & 7,60 & 1870,0 & 2283,0 & 4,17 & 6,38 & 744,7 & 760,0 \\
\hline 22 & 7,43 & 3,80 & 126,0 & 56,0 & 1,07 & 0,51 & 59,9 & 65,0 & 68 & 7,76 & 7,50 & 830,0 & 960,0 & 2,71 & 3,14 & 419,9 & 319,9 \\
\hline 23 & 5,84 & 7,40 & 64,0 & 301,0 & 1,34 & 0,62 & 14,9 & 215,0 & 69 & 6,94 & 7,20 & 1060,0 & 1082,0 & 2,99 & 2,50 & 474,9 & 490,1 \\
\hline 24 & 7,89 & 7,80 & 40,0 & 0,10 & 0,46 & 285,1 & 289,9 & 74,12 & $70^{* *}$ & 7,03 & - & 1410,0 & - & 2,93 & - & 614,8 & 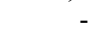 \\
\hline 25 & 7,98 & 8,00 & 405,0 & 394,0 & 0,44 & 0,05 & 299,9 & 295,0 & 71 & 7,20 & 7,50 & 4730,0 & 5060,0 & 2,40 & 3,34 & 2717,8 & 2913,9 \\
\hline 26 & 7,34 & 7,60 & 379,0 & 380,0 & 0,41 & 0,64 & 284,9 & 170,0 & 72 & 7,29 & 7,80 & 1300,0 & & 3,82 & 3,51 & 549,9 & 565,2 \\
\hline 27 & 8,77 & 6,90 & 771,0 & 7940,0 & 2,34 & 25,28 & 364,8 & 850,1 & 73 & 8,26 & 8,21 & 370,0 & 359,0 & 2,13 & 1,91 & 164,9 & 155,0 \\
\hline 28 & 7,56 & 8,10 & 410,0 & 0,73 & 0,51 & & 289,9 & 300,1 & 74 & 7,28 & 7,60 & 1400,0 & 135 & 3,58 & 2,79 & & 545,1 \\
\hline 29 & 7,37 & 8,90 & 70,0 & 53,0 & 0,54 & 0,28 & 44,9 & 50,0 & 75 & 7,95 & 7,70 & 1180,0 & 1328,0 & 3,65 & 0,04 & 459,9 & 590,1 \\
\hline 30 & 7,04 & 7,20 & 190,0 & 237,0 & 0,84 & 0,70 & 99,9 & 140,0 & 76 & 7,75 & 7,60 & 670,0 & 675,0 & 1,39 & 0,50 & 339,9 & 355,0 \\
\hline $31^{* *}$ & 7,99 & - & 660,0 & - & 1,68 & - & 374,9 & - & 77 & 7,36 & 7,90 & 950,0 & 822,0 & 1,82 & 1,43 & 589,9 & 580,2 \\
\hline 32 & 7,04 & 8,20 & 498,0 & 564,0 & 0,62 & 0,98 & 329,8 & 300,0 & 78 & 7,03 & 7,40 & 930,0 & 927,0 & 1,59 & 0,01 & 354,9 & 380,0 \\
\hline 33 & 7,75 & 8,00 & 627,0 & 778,0 & 1,29 & 2,20 & 379,8 & 355,0 & 79 & 7,39 & 7,90 & 710,0 & 710,0 & 1,02 & 0,61 & 369,9 & 425,1 \\
\hline 34 & 6,80 & 6,90 & 820,0 & 817,0 & 2,02 & 6,17 & 444,8 & 104,9 & 80 & 7,71 & 7,90 & 1350,0 & 1369,0 & 3,35 & 3,30 & 544,92 & 610,1 \\
\hline 35 & 5,47 & 6,10 & 100,0 & 133,0 & 0,73 & 1,65 & 29,9 & 44,9 & 81 & 7,53 & 7,90 & 1880,0 & 1714,0 & 3,57 & 5,26 & 869,4 & 680,23 \\
\hline 36 & 6,55 & 6,30 & 1090,0 & 1065,0 & 2,20 & 2,96 & 544,6 & 464,8 & 82 & 7,27 & 7,90 & 3130,0 & 3570,0 & 2,48 & 4,23 & 1754,0 & 1875,1 \\
\hline 37 & 7,09 & 7,50 & 1220,0 & 1052,0 & 3,85 & 5,25 & 494,9 & 355,0 & 83 & 7,10 & 7,50 & 1720,0 & 1851,0 & 1,96 & 2,60 & 1029,1 & 1044,8 \\
\hline $38^{* *}$ & 6,40 & - & 60,0 & - & 2,1 & - & 269,8 & & 84 & 7,39 & 8,00 & 1190,0 & 1444,0 & 2,90 & 5,14 & 619,7 & 520,1 \\
\hline 39 & 5,96 & 6,00 & 770,0 & 635,0 & 2,9 & 2,32 & 314,8 & 264,9 & $85^{* *}$ & - & 7,00 & - & 2105,0 & - & 3,56 & - & 915,0 \\
\hline 40 & 5,35 & 5,40 & 90,0 & 105,0 & 1,3 & 3,51 & 44,9 & 19,9 & $86^{* *}$ & - & 6,80 & - & 1644,0 & - & 0,29 & - & 904,6 \\
\hline $41^{* *}$ & 8,22 & - & 5370,0 & - & 10,9 & - & 1624,2 & & $87^{* *}$ & - & 6,70 & - & 3101,0 & - & 4,24 & - & 1459,6 \\
\hline 42 & 7,20 & 7,10 & 1140,0 & 1124,0 & 2,58 & 3,07 & 589,8 & 474,8 & 88 & 7,41 & 7,60 & 2470,0 & 2073,0 & 4,22 & 5,07 & 959,8 & 775,2 \\
\hline 43 & 7,26 & 7,30 & 610,0 & 649,0 & 1,57 & 1,83 & 344,9 & 275,0 & 89 & 7,28 & 7,80 & 710,0 & 640,0 & 2,18 & 1,95 & 364,8 & 385,0 \\
\hline 44 & 7,38 & 7,50 & 2580,0 & 1777,0 & 3,63 & 3,19 & 1229,8 & 695,1 & $90^{* *}$ & - & 7,70 & - & 3201,0 & - & 0,03 & - & 1814,3 \\
\hline 45 & 7,81 & 7,20 & 260,0 & 1101,0 & 0,65 & 2,03 & 169,9 & 564,9 & $91^{* *}$ & - & 7,30 & - & 1904,0 & - & 3,45 & - & 760,2 \\
\hline 46 & 7,87 & 7,60 & 260,0 & 256,0 & 0,55 & 0,84 & 179,9 & 169,9 & $92^{* *}$ & - & 8,10 & - & 568,0 & - & 0,74 & - & 365,1 \\
\hline
\end{tabular}


A.
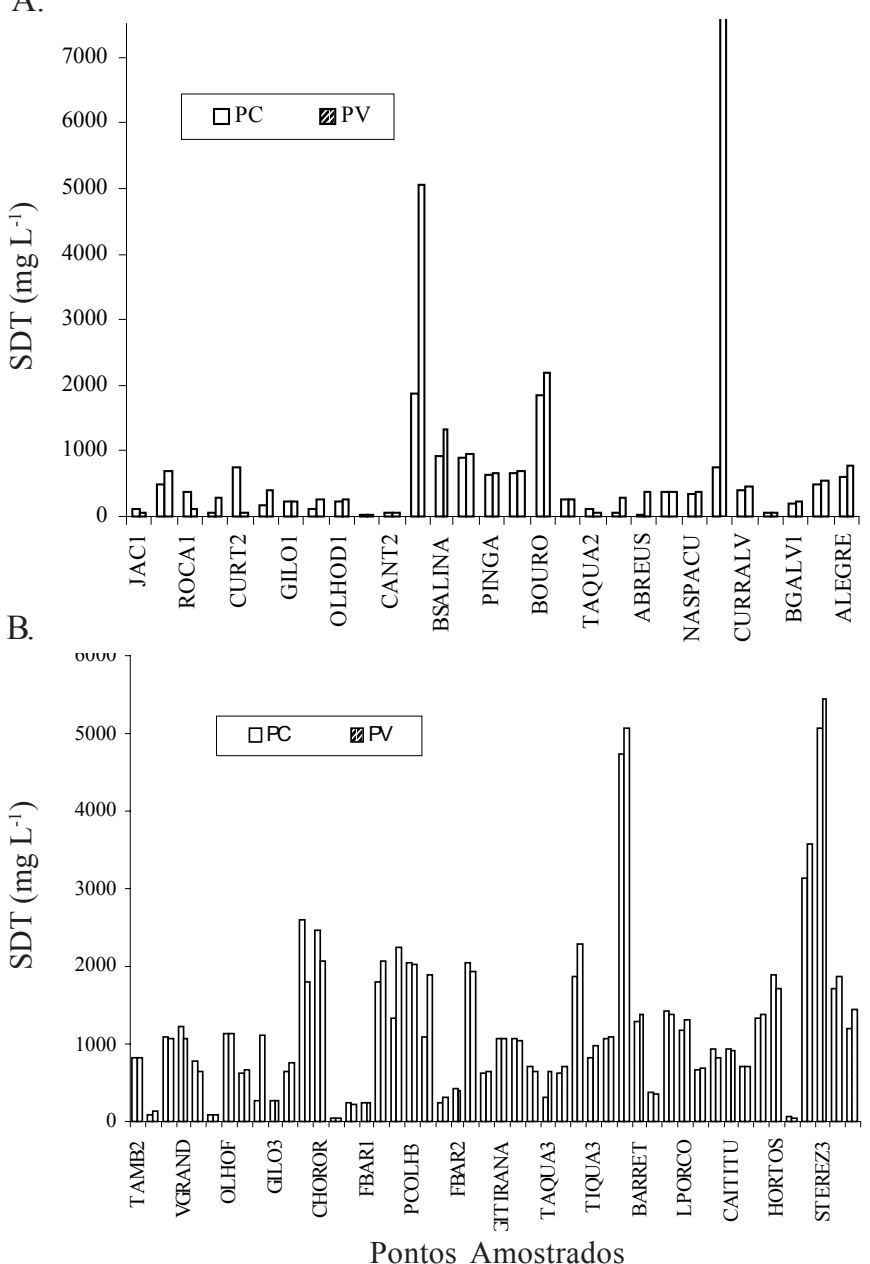

Figura 1. Variações espaço-temporais dos Sólidos Dissolvidos Totais (SDT) das águas superficiais (A) e subterrâneas (B) nos períodos de chuvas (PC) e sem chuvas (PV)

nas subterrâneas, cujos valores estão abaixo do limite recomendado pelo Ministério da Saúde $\left(500 \mathrm{mg} \mathrm{L}^{-1}\right)$ para a maioria das fontes (Tabela 1). A dureza é fator determinante na definição de uso das águas. Cerca de 57,57 e 44,83\% das fontes superficiais e 73,6 e 76,8\% das subterrâneas, nos respectivos períodos, são águas muito duras (DT $>200 \mathrm{mg} \mathrm{L}^{-1} \mathrm{de} \mathrm{CaCO}_{3}$, de acordo com a classificação de Custódio \& Llamas, citados por Santos (1997). Para uso doméstico, águas duras provocam maior consumo de sabão e dificultam o cozimento dos alimentos.

No contexto do uso da água para irrigação observa-se que a maioria das fontes superficiais não apresenta riscos de salinização, com apenas $35 \%$ delas classificadas como $\mathrm{C}_{3}$ e $\mathrm{C}_{4}$, porém 75,47 e $78,57 \%$ das fontes subterrâneas apresentam sérios riscos de salinização nos períodos analisados, sendo classificadas como $\mathrm{C}_{3}$ e $\mathrm{C}_{4}$.

Estudos realizados por Andrade \& Lopes (2000) sobre as águas subterrâneas da bacia hidrográfica do Rio Salitre, as classificam como muito duras e de elevada salinidade $\left(\mathrm{C}_{3}\right.$ e $\left.\mathrm{C}_{4}\right)$, além dos elevados valores de íons de carbonatos e cloretos, corroborando com esses resultados. Os riscos das águas promoverem a salinidade dos solos se tornam maiores quando os valores da condutividade elétrica da água de irrigação $\left(\mathrm{CE}_{\triangle}\right)$ são também maiores que $0,7 \mathrm{dS} \mathrm{m}^{-1}\left(\approx \mathrm{SDT}>450 \mathrm{mg} \mathrm{L}^{-1}\right)$ e do extrato de saturação do solo $\left(\mathrm{CE}_{\mathrm{S}}\right)$ maior que $4,0 \mathrm{dS} \mathrm{m}^{-1}(\approx \mathrm{SDT}$ $>2.500,0 \mathrm{mg} \mathrm{L}^{-1}$ ) (Ayers \& Westcot, 1991).

Nas áreas em que se pratica a irrigação intensiva, principalmente nas sub-bacias III e V (Figura 2), foram observadas variações significativas nas variáveis de qualidade das águas nos períodos analisados. Na região de Caatinga do Moura (sub-bacia III) foram notórios os problemas relacionados não apenas à quantidade mas, também, à qualidade das águas, gerando conflitos constantes e perdas da produção agrícola. A água utilizada para irrigação provém de poço jorrante (OLHDAG) e da barragem Airson Nolasco (OLHOD1), localizados à montante da área irrigada. As últimas fontes analisadas na seqüência foram a barragem Salinas (BSALINA) e o poço (FSUMI); nesta área obtiveram-se aumentos significativos nos valores de $\mathrm{pH}$, no período das chuvas, a medida em que se afasta das fontes de origem, ou seja, OLHOD1 $(\mathrm{pH}=6,35)$ e OLHDAG $(\mathrm{pH}=7,44)$, comparado com os valores de $\mathrm{pH}$ das parcelas irrigadas DENI1 $(\mathrm{pH}=7,98)$ e AIFINAL $(\mathrm{pH}=8,30)$; esses aumentos estão relacionados às atividades agrícolas implementadas na área.

Entre as barragens OLHOD1 e BSALINA, pontos extremos desta área, observam-se aumentos significativos nos valores $\operatorname{dos} \operatorname{SDT}\left(271,0 ; 1.317,0 \mathrm{mg} \mathrm{L}^{-1}\right)$, RAS $\left(1,83 ; 3,90 \mathrm{mmol} \mathrm{L}^{-1}\right)^{1 / 2}$, DT $\left(120,0 ; 610,0 \mathrm{mg} \mathrm{L}^{-1}\right)$ no período sem chuvas, considerado crítico com relação à disponibilidade de água, tanto que não havia água para irrigar. No período das chuvas a água nas parcelas irrigadas (DENI1 e AIFINAL) apresentou maior

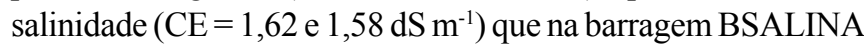
$\left(\mathrm{CE}=1,38 \mathrm{dS} \mathrm{m}^{-1}\right)$, o mesmo ocorrendo com os poços OLHDAG $\left(\mathrm{CE}=0,34 \mathrm{dS} \mathrm{m}^{-1}\right)$ e FSUMI $\left(\mathrm{CE}=3,10 \mathrm{dS} \mathrm{m}^{-1}\right)$. Nesta região, os riscos de sodicidade são maiores pelo fato do solo ser pesado ( $50 \%$ de silte e argila), conter alto teor de matéria orgânica (31 $\mathrm{g} \mathrm{dm}^{-3}$ ) e apresentar drenagem deficiente, com sinais evidentes de salinização. Também, o método de irrigação por superfície contribui para elevar a salinidade.

$\mathrm{Na}$ área irrigada com água da fonte BARRET1 (sub-bacia IV), classificada como $\mathrm{C}_{4} \mathrm{~S}_{2}\left(\mathrm{CE}_{\mathrm{A}}=7,0\right.$ e $\left.7,17 \mathrm{dS} \mathrm{m}^{-1}\right)$, além dos elevados valores dos SDT, DT e $\mathrm{Cl}$, a condutividade elétrica do extrato de saturação do solo também foi elevada $\left(\mathrm{CE}_{\mathrm{S}}=31,4\right.$ $\mathrm{dS} \mathrm{m} \mathrm{m}^{-1}$ ), com uma PST $=10,63 \%$, classificando-o como solo salino (Tabela 2). Esta água está sendo usada para irrigar a cultura do mamoeiro (Carica papaya), que é moderadamente tolerante à salinidade (Ayers \& Westcot, 1991). O sistema de irrigação usado é por superfície, o qual favorece a maior acumulação de sais no solo devido à baixa eficiência de aplicação de água. De modo geral, as culturas se comportam de formas diferentes em condições de salinidade; culturas mais sensíveis, altas concentrações de sais na água de irrigação têm reflexos negativos, tanto no desenvolvimento das plantas como na produtividade e qualidade dos produtos, manifestando-se desde as fases de germinação e emergência das plântulas até a fase produtiva.

Aumentos nas variáveis de qualidade das águas em áreas sob influência da irrigação resultam da baixa eficiência dos sistemas de produção, envolvendo não apenas o manejo da água de irrigação e do solo, mas também dos agroquímicos aplicados; portanto, há necessidade de um manejo adequado desses fatores de produção, de forma a garantir a sua 


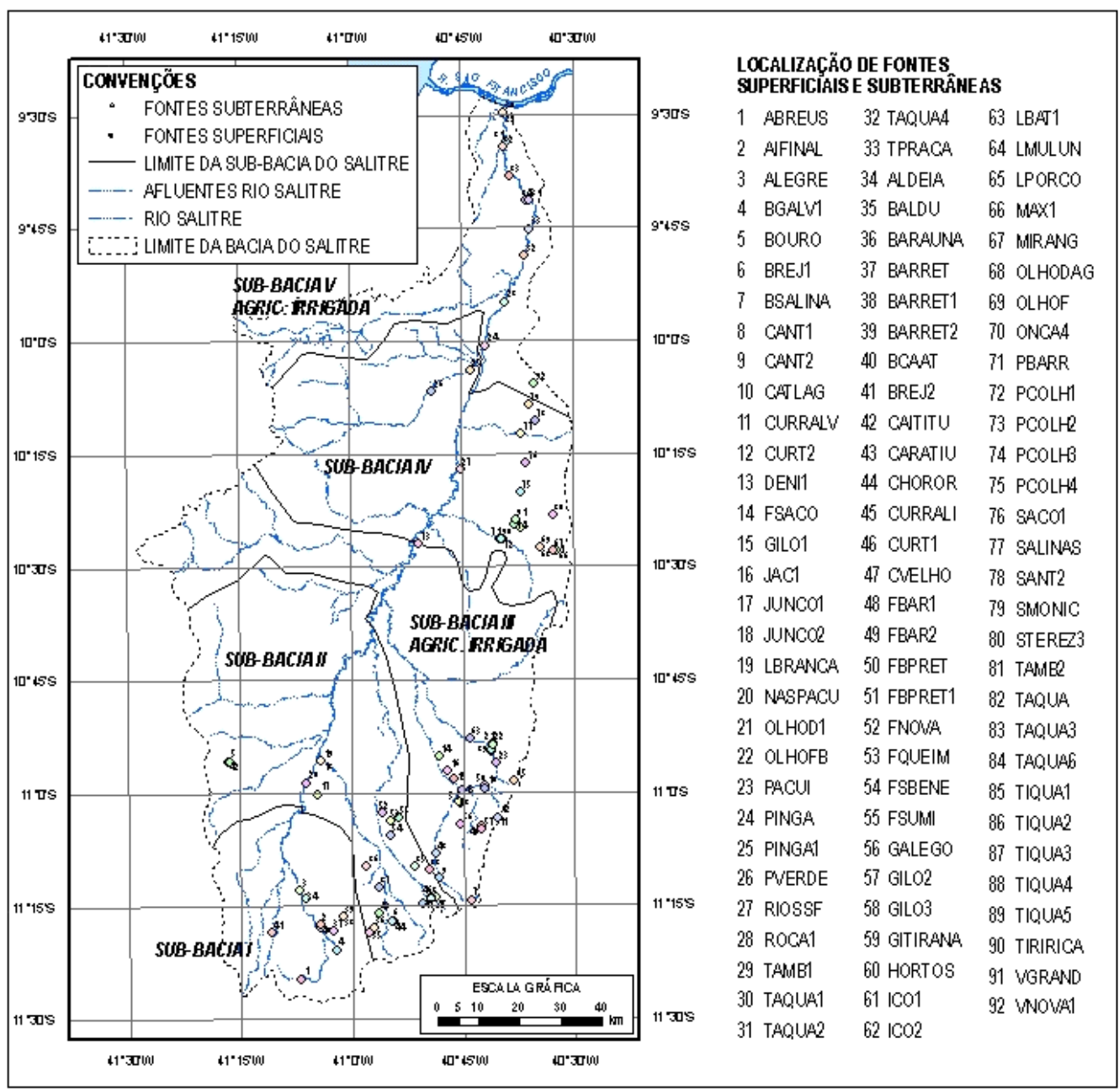

Figura 2. Localização das fontes hídricas superficiais e subterrâneas analisadas na bacia do Salitre, identificando-se a área irrigada

Tabela 2. Características físico-químicas e classificação dos solos quanto aos riscos de salinidade e sodicidade da bacia hidrográfica do Rio Salitre, no período sem chuvas

\begin{tabular}{|c|c|c|c|c|c|c|c|c|c|c|c|}
\hline \multirow{2}{*}{ Código } & \multirow{2}{*}{$\begin{array}{c}\mathrm{CE}_{\mathrm{s}} \\
\left(\mathrm{dS} \mathrm{m}^{-1}\right)\end{array}$} & \multirow[t]{2}{*}{$\mathrm{pH}$} & \multirow{2}{*}{$\begin{array}{l}\text { M.O. } \\
\left(\mathrm{g} \mathrm{dm}^{-3}\right)\end{array}$} & \multirow{2}{*}{$\begin{array}{c}\mathrm{P} \\
\left(\mathrm{mg} \mathrm{dm^{-3 }}\right)\end{array}$} & K & $\mathrm{Ca}$ & $\mathrm{Mg}$ & $\mathrm{Na}$ & $\mathrm{H}+\mathrm{Al}$ & \multirow{2}{*}{$\begin{array}{l}\text { PST } \\
(\%)\end{array}$} & \multirow{2}{*}{$\begin{array}{c}\text { Classes } \\
\text { Salinidade }\end{array}$} \\
\hline & & & & & \multicolumn{5}{|c|}{$\left(\mathrm{mmol}_{\mathrm{c}} \mathrm{dm}^{-3}\right)$} & & \\
\hline 1.JAC1 & 0,53 & 5,9 & 16,7 & 3,0 & 0,33 & 1,3 & 1,1 & 0,02 & 3,46 & 0,32 & Normais \\
\hline 10.OLHOD1 & 1,38 & 8,7 & 23,9 & 8,0 & 0,29 & 4,8 & 4,9 & 2,73 & 0,00 & 21,46 & Sódicos \\
\hline 15.DENI1D & 8,54 & 8,3 & 31,8 & 28,0 & 1,13 & 12,0 & 3,7 & 3,14 & 0,00 & 15,72 & Salino-sódicos \\
\hline 16.DENI1 & 17,88 & 8,2 & 34,4 & 35,0 & 1,80 & 12,8 & 8,8 & 0,35 & 0,00 & 1,47 & Salinos \\
\hline 49.LBAT 1 & 0,45 & 5,4 & 31,4 & 6,0 & 0,25 & 1,0 & 0,9 & 0,98 & 6,75 & 9,92 & Normais \\
\hline 17.PVERDE & 0,93 & 8,7 & 12,5 & 83,0 & 0,87 & 7,0 & 2,7 & 0,27 & 0,00 & 2,49 & Normais \\
\hline 23.TAQUA4 & 10,2 & 9,1 & 11,4 & 79,0 & 0,22 & 3,4 & 3,1 & 3,04 & 0,00 & 31,15 & Salino-sódicos \\
\hline 21.TAQUA1 & 0,62 & 8,7 & 11,0 & 75,0 & 0,85 & 3,7 & 2,4 & 0,04 & 0,00 & 0,57 & Normais \\
\hline 22.TAQUA2 & 1,09 & 7,5 & 6,8 & 14,0 & 1,07 & 2,7 & 2,2 & 0,16 & 0,00 & 2,61 & Normais \\
\hline 73.BCAAT & 43,1 & 7,8 & 36,9 & 7,0 & 0,92 & 11,5 & 24,8 & 3,56 & 0,00 & 8,73 & Salinos \\
\hline 73.BCAATS & 0,42 & 8,4 & 25,0 & 115,0 & 1,01 & 8,0 & 2,4 & 0,35 & 0,00 & 2,98 & Normais \\
\hline 71.BARET1 & 31,4 & 7,6 & 17,2 & 17,0 & 1,13 & 17,3 & 7,3 & 3,06 & 0,00 & 10,63 & Salinos \\
\hline 25.PACUI & 0,28 & 8,1 & 18,8 & 27,0 & 1,17 & 11,3 & 2,7 & 0,07 & 0,00 & 0,46 & Normais \\
\hline 82.STEREZ1 & 0,47 & 7,2 & 13,3 & 29,0 & 0,89 & 8,2 & 5,7 & 3,06 & 0,00 & 17,14 & Sódicos \\
\hline 30.BGALV1 & 1,09 & 8,2 & 16,1 & 13,0 & 0,66 & 11,9 & 4,0 & 2,80 & 0,00 & 14,46 & Salinos \\
\hline 32.GOIAB & 6,93 & 8,5 & 29,9 & 3,0 & 0,57 & 7,1 & 7,4 & 0,08 & 0,00 & 0,53 & Salinos \\
\hline
\end{tabular}

sustentabilidade, visando a obtenção de elevadas produtividades (Brito, 2003).

Variabilidades nos parâmetros analisados também foram observadas nas características físico-químicas dos solos das áreas irrigadas. Na área da fonte LBAT1, os solos são arenosos, ácidos $(\mathrm{pH}=5,4)$ de baixa fertilidade que, se explorados com culturas de maior rentabilidade econômica, necessitarão de correção do pH, uma vez que nestas condições elementos como 
Tabela 3. Classificação geoquímica das águas superficiais e subterrâneas da bacia hidrográfica do Salitre

\begin{tabular}{|c|c|c|c|c|c|c|c|c|}
\hline \multirow{2}{*}{ Classes } & \multicolumn{2}{|c|}{ Total } & \multicolumn{2}{|c|}{$\mathrm{Ca}-\mathrm{Mg}-\mathrm{Na}$} & \multicolumn{2}{|c|}{$\mathrm{Ca}-\mathrm{Na}^{*}$} & \multicolumn{2}{|c|}{ Outros } \\
\hline & Amostras & $(\%)$ & Amostras & $(\%)$ & Amostras & $(\%)$ & Amostras & $(\%)$ \\
\hline \multicolumn{9}{|c|}{ Fontes superficiais } \\
\hline Bicarbonatadas & 4 & 16,7 & 3 & 12,5 & - & - & 1 & 4,2 \\
\hline Bicarbonatadas Cloretadas & 18 & 75,0 & 13 & 54,2 & 2 & 8,3 & 3 & 12,5 \\
\hline Bicarbonatadas Sulfatocloretadas & 2 & 8,3 & 1 & 4,2 & 1 & 4,2 & - & - \\
\hline Sub-Total & 24 & 82,7 & 17 & 70,8 & 3 & 12,5 & 4 & 16,7 \\
\hline Cloretadas & 5 & 17,2 & 2 & 40,0 & - & - & 3 & 60,0 \\
\hline Total & 29 & 100 & 19 & 65,5 & 3 & 10,3 & 7 & 24,1 \\
\hline \multicolumn{9}{|c|}{ Fontes subterrâneas } \\
\hline Bicarbonatadas & 5 & 15,1 & 5 & 15,1 & - & - & - & - \\
\hline Bicarbonatadas-Cloretadas & 28 & 84,8 & 21 & 63,6 & 3 & 9,1 & 4 & 12,1 \\
\hline Sub-Total & 33 & 58,9 & 26 & 78,8 & 3 & 9,1 & 4 & 12,1 \\
\hline Cloretadas & 23 & 41,0 & 18 & 78,3 & 2 & 8,7 & 3 & 12,1 \\
\hline Total & 56 & 100 & 44 & 78,7 & 5 & 8,9 & 7 & 12,5 \\
\hline
\end{tabular}

Al, Fe e Mn são solúveis e podem permanecer no solo ou serem transportados pelas águas, para as fontes hídricas (Primavesi, 1988). Nas demais áreas, os solos tendem à alcalinidade, com valor máximo na área TAQUA5 $(\mathrm{pH}=9,1)$, apresentam elevados teores de cálcio, magnésio e fósforo (Tabela 2). O cálcio, além de nutriente essencial ao desenvolvimento das plantas, tem também a função de corrigir o $\mathrm{pH}$ dos solos, neutralizar a toxicidade de alguns elementos (Al, Mn, Na e Mg) e reduzir a relação de adsorção de sódio (RAS), tanto da água de irrigação quanto do solo, contribuindo para uma agregação melhor de suas partículas (Primavesi, 1988; Ayers \& Westcot, 1991). Esta diversidade de condições edáficas impõe a definição de medidas diferenciadas quanto ao manejo sustentável dos solos dessas áreas.

Resultados das análises de solos indicam a presença de solos sódicos (OLHOD); salino-sódicos (DENI1D, TAQUA5) e salinos (DENI1), embora medidas de recuperação dos solos tenham sido implementadas (Tabela 2). Esses solos são classificados como Cambissolos (DENI, AIFINAL, CURRALV, GOIAB e ALEGRE), Litólicos (TAQUA, TAQUA1 e TAQUA2) e Vertissolos (HORTOS e STEREZ). Segundo Leprun (1983), trata-se de solos ricos em cátions trocáveis e águas classificadas, quanto à salinidade, acima de $\mathrm{C}_{2} \mathrm{~S}_{2}$ não devem ser utilizadas, pois promovem sua salinidade; portanto, nestas condições deve-se aplicar lâminas de lixiviação e usar drenagem que podem reduzir os riscos de salinidade dos solos (Ayers \& Westcot, 1991).

Em geral, elevada salinidade das águas, altos valores de dureza total e de cloretos, está associada à sua composição geoquímica. Cerca de 82,7 e $58,9 \%$ das fontes superficiais e subterrâneas, foram classificadas como águas bicarbonatadas, das quais 70,8 e 78,8\% são cálcicas-magnesianas-sódicas e, 17,2 e $41,0 \%$, como as águas cloretadas, respectivamente (Tabela 3). Águas bicarbonatadas usadas para irrigação, principalmente em regiões áridas e semi-áridas, devido às elevadas taxas evapotranspirométricas, favorecem na redução da água no solo e aumentam a concentração salina da solução do solo, de maneira que os íons de $\mathrm{Ca}^{++}$e $\mathrm{Mg}^{++}$alcançam os limites de solubilidade e precipitam, aumentando a RAS.
Auzmendi et al. (2002) em estudos realizados sobre a qualidade das águas subterrâneas, em Irecê, BA, onde predominam rochas do Grupo Bambuí, semelhante às da bacia em estudo obtiveram, também, elevados valores nos SDT, $\mathrm{Cl}$, $\mathrm{HCO}_{3}$ e associaram os resultados aos fatores litológicos, climáticos e antrópicos.

\section{CONCLUSÕES}

1. Não se observaram variações significativas nas variáveis de qualidade das águas entre os períodos de chuva e sem chuva, porém se notou que nas áreas sob influência da irrigação ocorreram aumentos significativos nas variáveis, principalmente no $\mathrm{pH}$, na condutividade elétrica da água e do extrato de saturação do solo.

2. De modo geral, a qualidade das águas da bacia hidrográfica do Rio Salitre, com elevados valores de SDT, DT, $\mathrm{Cl}^{-}, \mathrm{HCO}_{3}^{-}, \mathrm{Ca}^{++}$, se deve a fatores naturais, principalmente climáticos e geológicos, observando-se que $82,7 \%$ e $58,9 \%$ das fontes superficiais e subterrâneas, respectivamente, foram classificadas como bicarbonatadas.

3. Esses resultados apontam para a necessidade de medidas de preservação e conservação dos recursos hídricos e dos solos, como adequado manejo do sistema solo-água-planta, de forma a reduzir os riscos de salinização dos solos e das águas dessa bacia hidrográfica para permitir a sustentabilidade ambiental.

\section{LITERATURA CITADA}

Andrade, L.N.V. de; Lopes, H.L. Aplicação de modelos hidrológicos superficiais e subsuperficiais em bacias hidrográficas: Fase I - compilação do banco de dados. In: Congresso Brasileiro de Cadastro Técnico Multifinalitário, 2000, Florianópolis. Anais... Florianópolis: UFSC, 2000. p.125 . 
Auzmendi, I.A.; Evangelista, I.M.; Silva, H.P. Síntesis de los conocimientos sobre la hidrogeología de la cuenca del rio Verde e Jacaré, Irecê-BA, Brasil: In: Seminário Internacional CYTED-XVII, 2., 2002, Salvador,BA. Resumos... Salvador: CYTED/UFBA/UEPS/SRH-BA/MMA-SRH/FAPEX, 2002. n.p.

Ayers, R.S.; Westcot, D.W. A qualidade da água na agricultura. Campina Grande: UFPB, 1991. 218p. FAO: Irrigação e Drenagem, 29. Revisado 1

Bahia. Secretaria doPlanejamento, Ciência e Tecnologia. Avaliação dos recursos hídricos em bacias hidrográficas do Estado da Bahia: Bacia do Rio Salitre. Salvador, 1986. 2v.....p.

Brasil. Ministério da Saúde. Portaria n ${ }^{\circ} .518$, de 25 de março de 2004. Diário Oficial, Brasília, 26 de março de 2004. Seção 1, $266 \mathrm{p}$.

Brito, L.T. de L. Avaliação de impactos das atividades antrópicas sobre os recursos hídricos da bacia hidrográfica do rio Salitre, BA, e classificação das fontes hídricas. Campina Grande:UFCG, 2003. 184p. Tese Doutorado

Brown, L.; Flavin, C.; French, H. Estado do mundo 2000. Tradução H. Mallett. Salvador: UMA Editora, 2000. 288p.

Claessen, M. E. C. (Org.). Manual de métodos de análise do solo. 2.ed. rev. atual. Rio de Janeiro: EMBRAPA-CNPS, 1997. 212p. Documentos, 1

Freitas, M.A.V. de; Santos, A.H.M. Importância da água e da informação hidrológica. In: Freitas, M.A.V. de. (Ed.). O estado das águas no Brasil; perspectivas de gestão e informações de recursos hídricos. Brasília: ANEEL/MME/ MMA-SRH/OMM, 1999. p.13-16.
Girardin, P.; Rockstaller, C.; Werf, H. van der. Assessment of potential impacts of agricultural practices on the environment: the AGRO*ECO method. Environmental Impact Assessment Review, New York, v.20, p.227-239, 2000.

Leal, A. de S. As águas subterrâneas no Brasil: ocorrências, disponibilidade e usos. In: Freitas, M.A.V. de. (Ed.). O estado das águas no Brasil; perspectivas de gestão e informações de recursos hídricos. Brasília: ANEEL-SRH/MME/MMASRH/OMM, 1999. p.139-164.

Leprun, J.C. Relatório de fim de convênio de manejo e conservação do solo no Nordeste brasileiro, Recife: SUDENE. 1983. 290p. 5 mapas anexo.

Neves, B.B. de B. Inventário hidrogeológico básico do Nordeste: Folha 24 - Aracajú-SE. Recife:SUDENE. 1972. 284p. Hidrogeologia, 26.

Ongley, E.D. Controle da poluição da água pelas atividades agrícolas. Campina Grande: UFPB, 2001. 92p. FAO. Irrigação e Drenagem, 55

Pizarro, F. Drenaje agricola y recuperacion de suelos salinos. Madrid: Editora Agrícola Española, 1978. 521p.

Primavesi, A. Manejo ecológico do solo: a agricultura em regiões tropicais. 9.ed. São Paulo: Nobel, 1988. 549p.

Resende, A.V. de; Agricultura e qualidade da água: contaminação da água por nitrato. Brasília: EMBRAPA Cerrados. 2002.29p. Documentos 57.

Santos, A.C. Noções de hidroquímica. In: Feitosa, F.A.C.; Manoel Filho, J. Hidrogeologia: conceitos e aplicações. Recife: CPRM/LABHID. 1997. cap.5, p.81-108.

SEI - Superintendência de Estudos Econômicos e Sociais da Bahia. Balanço hídrico do Estado da Bahia. Salvador, 1999. 250p. SEI. Série Estudos e Pesquisa, 45 